\title{
PNEUMATOSIS INTESTINALIS WITH PNEUMOPERITONEUM MISDIAGNOSED AS ACUTE ABDOMEN- A CASE REPORT
}

\author{
Y. Georgiev*, G. Zafirov, Y. Dimcheva, Z. Rusinova, A. Karashmalakov \\ Surgery Department, Virgin Mary Hospital, Burgas, Bulgaria
}

\begin{abstract}
Background: Pneumatosis intestinalis (PI), is defined as the presence of intramural gas within the bowel wall. Pneumatosis intestinalis is not a diagnosis. It is more a histological finding or a radiology sign, rather than a disease on itself. In most of the cases it is a benign finding, but it may be a reason for an unnecessary laparotomy, especially when associated with pneumoperitoneum.

Case report: We present a case of pneumatosis intestinalis in a patient with a chronic obstructive pulmonary disease misdiagnosed as intestinal perforation due to bowel ischemia. The patient was admitted with increasing abdominal pain and nausea. On examination the abdomen was tender with diffuse pain. Plain X-rays and CT scans showed pneumoperitoneum with intramural gas in small intestine's wall. With preoperative diagnosis of bowel ischemia the patient underwent exploratory laparotomy, on which a benign PI was found.

Conclusion: Carefully taken medical history, prompt exam, laboratory and instrumental data all should be carefully analyzed before a decision for emergency operation is done. It is important to divide PI in two categories- benign and life-threating.
\end{abstract}

Key words: intramural gas, pneumoperitoneum, gas cysts

\section{INTRODUCTION}

Duvernoy first described pneumatosis intestinalis (PI) in 1754 [1]. Since then, numerous case reports and reviews have appeared in the world literature. PI is more common in the small intestine $(42 \%)$ than the colon $(36 \%)$, with involvement of both in $22 \%$ [2].While PI in most of the cases is benign condition, up to $27 \%$ of patients with benign forms of pneumatosis may be misdiagnosed with surgical abdomens, and will undergo unnecessary laparotomy [3]

The presence of free gas in the abdomen with intramural bowel gas is always alerting sign. It is important to divide PI in two categoriesbenign and life-threating. Clinical examination and laboratory data are mandatory for the decision in which category the PI will be included and respectively treated.

\section{CASE PRESENTATION}

A 88 year old caucasian male was admited to the surgery ward with increasing abdominal

\footnotetext{
*Correspondence to: Yonko Petrov Georgiev M.D. - Surgery Department, Virgin Mary Hospital, Burgas, A. Stamboliiski Str. Bulgaria, e-mail: yonko_georgiev@abv.bgtel : +359899127849
}

pain and naussea for less than 24 hours. On physical examination patient was stable hemodinamically, abdomen was tender, with diffuse abdominal pain and very silent bowel signs. Co-morbidity of the patient included chronic obstructive pulmonary disease, arterial hypertony and a survived brain stroke. The plain $\mathrm{X}$ ray of the abdomen showed free gas in the abdominal cavity, further examination included abdominal CT which confirmed the free gas and showed typical linear pneumatosis intestinalis of the small bowel. No gas in the portal vein was observed. As additional finding there was an old parasytic cyst ot the liver. Laboratory data showed elevated CRP, but no increased white blood count. With preoperative diagnosis of intestinal ischemia the patient underwent emargency laparotomy. Intraoperative findings included thickened wall ot the small intestine, due to the intramural gas, mesocolic lymphadenomegaly and a non-vital parasytic liver cyst. No signs of bowel ischemia or perforation of holow viscera was observed so the abdomen was closed. The postoperative period was uneventful. On postoperative follow up patient has no complaints. 


\section{DISCUSSION}

PI is a rare condition usually caused by an underlying condition. The overall incidence is unknown due to the asymptomatic course of the disease in most cases. Although PI can be seen on abdominal radiographs, CT is the most sensitive imaging test for identification of PI. The CT detection of PI appears to be increasing, likely as a consequence of increased use of CT [4]

When assessing the radiographic appearance of intramural gas, the organization and distribution of the gas in the bowel wall is diagnostically helpful. As stated by Heng, typical patterns of intramural gas include microvesicular, cystic and linear variants [5].

Microvesicular pneumatosis is associated with invasive procedures like colonoscopies and/or polypectomies and is generally clinically and radiographically occult.It is a histological diagnosis due to the fact the air collections rarely exceed 100 micrometers in diameter and cannot be seen on plain abdominal X-rays.

The cystic variant of PI is characterized by macroscopic submucosal cysts which may vary in size. These protrude into the bowel lumen and often are mistaken as poliposis coli [6]. It is now known that PI is associated with various conditions including COPD, immunosupression therapy, immunocompromise, steroid usage, and IBD[7] When such cyst rupture they cause a sterile pneumoperitoneum whithout peritonitis.[1] Jamart from 919 cases conclude that small bowel PI is associated with pneumoperitoneum in about $10 \%$, while free air in the abdomen is seen in about $2 \%$ in those with large bowel PI[3]. The cystic variant of pneumatosis intestinalis is nonspecific and in more of the cases benign [8] .

Linear pneumatosis is probably the most alerting sign and is associated with serious
GEORGIEV Y., et al.

abdominal conditions such as abdominal ischemia, infarction and necrotizing eneterocolitis in infants. However, this variant can be a benign finding too. Linear PI is characterized by a linear pattern of the intramural gas that is oriented parallel to the bowel wall. Once observed linear PI it is mandatory to search for portal vein gas prognostic sign of bowel ischemia.

The origin of the air in the bowel wall is still unclear. There are two major theories mechanical and bacterial. The first one hypothesizes that gas enters into the bowel wall from either the intestinal lumen or the lungs via the mediastinum due to some mechanism causing increased pressure (i.e., bowel obstruction or emphysema). A bacterial theory proposes that gas-forming bacteria enter the submucosa through mucosal rents or increased mucosal permeability and produce gas within the bowel wall. [9]. Interesting fact is that gas collections in the bowel wall may consis of up to $50 \%$ hydrogen, which is a product of bacterial metabolism, and is not specific for humans [10]. However pneumoperitoneum from PI does not cause peritonitis even left for a long time [1].

The presence of free air in the abdominal cavity is always an alarming sign. Carefully taken medical history and a prompt examination are mandatory for the decision to operate. Laparotomy should not be made only on the basis of radiographic findings alone. Pneumoperitoneum is not necessarily caused by bowel perforation but may represent a ruptured cyst. Once PI is diagnosed further examination is needed to exclude unnecessary operation. Schröpfer recommend a standardized algorithm in case of PI [10] With laboratory findings of elevated CRP or white blood cell count, as well as signs of sepsis, bowel perforation or free gas in the portal vein immediate surgery is indicated.

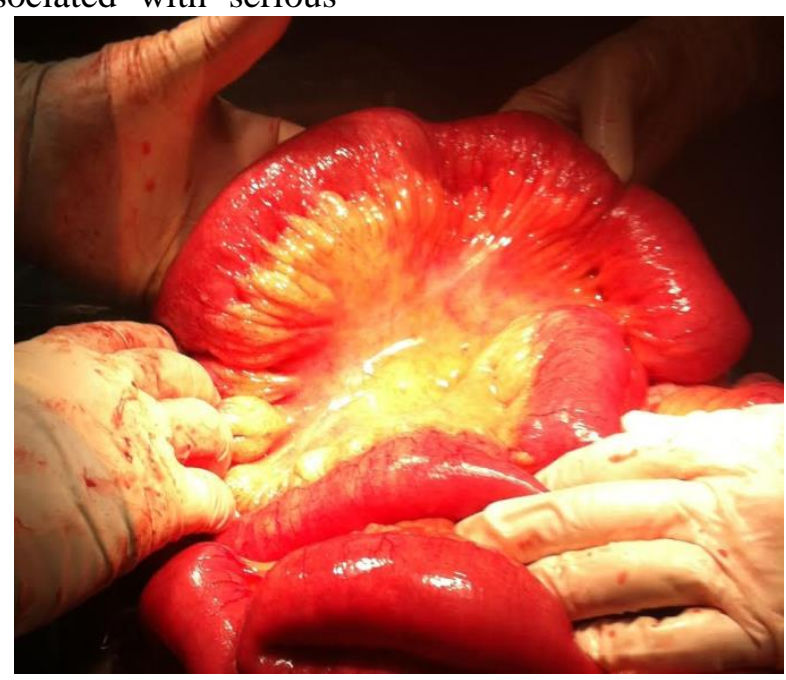




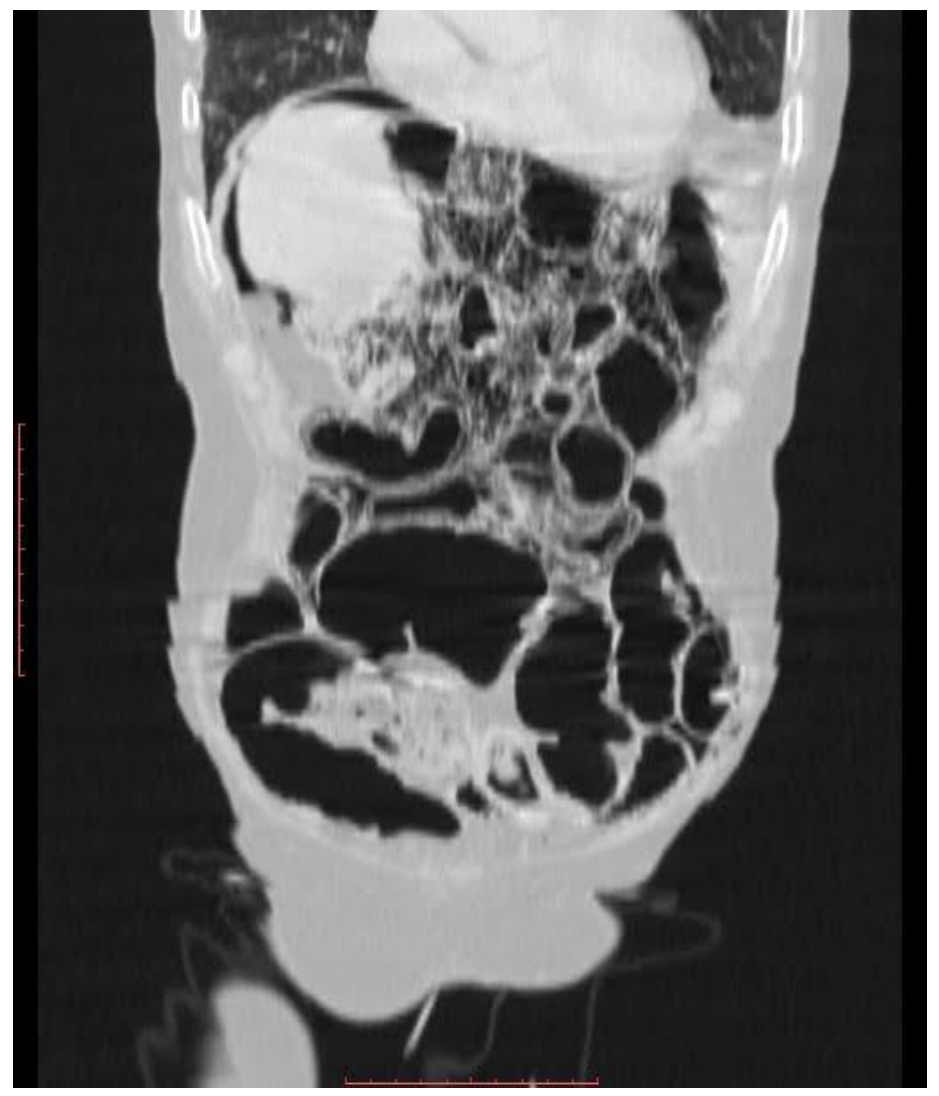

GEORGIEV Y., et al.

Figure 1 \& 2. Preoperative CT scans showing linear pattern of intramural bowel gas and pneumoperitoneum

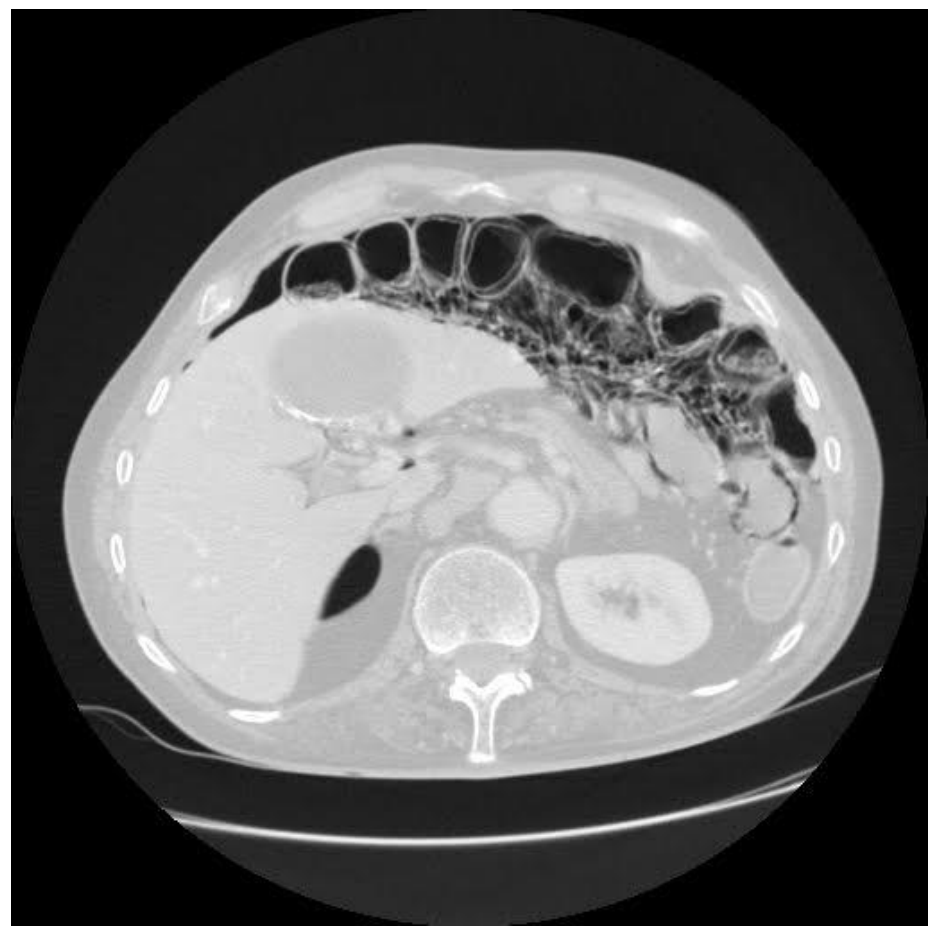

Figure 3. Intraoperative appearance of benign PI

\section{CONCLUSION}

$\mathrm{PI}$ is a rare condition, which nowadays is more often recognized due to the fact of increased use of advanced imaging technologies and especially CT. The presence of intramural bowel gas and pneumoperitoneum is not an indication for emergency operation especially in a comorbid patient in whom the underlying condition is the reason for development of PI. Carefully taken medical history, prompt exam, laboratory and instrumental data all should be carefully 
analyzed before a decision for emergency operation is done.

\section{ACKNOWLEDGEMENTS}

Authors declare that there is no financial or other conflict of interest.

Informed Consent: Written informed consent was obtained from the patient for the publication of this case report and any accompanying images.

\section{REFERENCES}

1. Koss LG. Abdominal gas cysts (pneumatosis cystoides intestinorum hominis): an analysis with a report of a case and a critical review of the literature. Arch Pathol 1952; 53:523-549

2. C. Braumann, C. Menenakos, and C. A. Jacobi, "Pneumatosis intestinalis - a pitfall for surgeons?" Scandinavian Journal of Surgery, vol. 94, no. 1, pp. 47-50, 2005

3. Jamart J. Pneumatosis cystoides intestinalis. A statistical study of 919 cases. Acta Hepato Gastroenerol 1979; 26:419-22.

4. Neumayer L, Wako E, Fergestaad J, Dayton M. Impact of journal articles and grand rounds on practice: CT scanning in
GEORGIEV Y., et al. appendicitis. J Gastrointest Surg 2002; 6:338-341

5. Heng Y, Schuggler MD, Haggitt RD, Rohrmann CA. Pneumatosis intestinalis: a review. Am J Gastro 1995;90(10):1747-58

6. Yilin Vogel et al. Pneumatosis cystoides intestinalis of the ascending colon related to acarbose treatment: a case report. Journal of Medical Case Reports 2009, 3:9216 doi: 10.4076/1752-1947-3-9216

7. Holt S, Stewart I, Heading R. Resolution of primary pneumatosis coli. J R Coll Surg 1979;23:297-9

8. David M. Liu et al. Benign pneumatosis intestinalis: a cause of massive pneumoperitoneum in the adult. Can $\mathbf{J}$ Emerg Med 2003;5(6):416-20

9. Galandiuk S, Fazio VW. Pneumatosis cystoides intestinalis: a review of the literature. Dis Colon Rectum 1986; 29:358363

10.Engelbert Schröpfer , Thomas Meyer. Surgical aspects of pneumatosis cystoides intestinalis: two case reports. Cases Journal 2009, 2:6452 doi: 10.4076/1757-1626-26452 